Revue critique

\title{
Éléments pour une histoire critique des pratiques de dépistage : le cas de la France
}

\author{
Elements for a critical history of screening practices: the case of France
}

Jacques RODRIGUEZ ${ }^{1}$

${ }^{1}$ Professeur de sociologie, Université de Lille-SHS

\section{MOTS CLÉS \\ Dépistage, Prévention, Politiques sanitaires, France, Sociohistoire.}

\section{KEY WORDS}

Screening, prevention, health policy, France, social history.

\section{Résumé}

Le dépistage est une pratique qui, en France, est souvent discutée ou évaluée par les acteurs du monde médical ; elle est abordée aussi par les chercheurs en sciences sociales, qui étudient les conditions de sa mise en œuvre, ses effets, ou encore ses dérives éventuelles. Dans une perspective sociohistorique, on se propose ici d'opérer un décentrement en s'interrogeant plutôt sur les transformations successives de cette pratique. Pour ce faire, on insistera sur le dépistage de la tuberculose, du cancer et du sida afin de faire apparaître les inflexions survenues depuis la fin du 19ème siècle et de souligner la radicale nouveauté de l' «État dépisteur » contemporain.

\begin{abstract}
In France, screening is a practice often discussed and evaluated by the actors of the medical world; social scientist also address it, they study conditions for its implementation, its effects, or its possible deviations. From a social historical perspective, it is proposed here to operate a decentration by questioning rather the successive transformations of this practice. Tuberculosis, cancer and AIDS will be emphasized in order to reveal the inflexions that have occurred since the end of the 19th century and to underline the radical newness of the contemporary scouting state.
\end{abstract}

\section{Introduction}

Nous sommes tous de plus en plus concernés - et cernés - par les dépistages. Cette pratique de santé rencontre en effet un succès grandissant et, dans de très nombreux pays, les individus sont soumis à des examens de dépistage dès leur conception, puis encore à la naissance, et tout au long de la vie à la faveur de diverses campagnes de prévention. Sans compter que les dépistages se multiplient d'autant plus vite qu'ils débordent du monde médical où ils ont été forgés: l'institution judiciaire, la police ou l'administration sociale recourent également à ce procédé [1]. Un tel foisonnement

Auteur correspondant: jacques.rodriguez@univ-lillez.fr 
est d'autant plus remarquable que le dépistage est un procédé très ambivalent qui, à la fois, fascine et inquiète : d'un côté, il permet a priori de prendre le mal à la racine, donc de le prendre de vitesse ; mais d'un autre côté, il suscite des réserves, des résistances et même des polémiques. D'aucuns s'interrogent par exemple sur l'efficacité, l'utilité, voire l'innocuité de certaines opérations de dépistage, tandis que d'autres soulignent avec méfiance les enjeux économiques d'une pratique qui contribue à élargir le marché de la prescription et des appareillages. Comment, dès lors, peut-on comprendre l'extension et la banalisation d'une pratique aussi ambiguë et controversée ? Celle-ci constitue sans doute une réponse au désir de santé que partagent nombre d'individus, lesquels, souvent, " ne veulent plus attendre d'être malades ", ni attendre les premiers symptômes pour être traités [2]. Mais on peut aussi faire l'hypothèse que la success story du dépistage nous éclaire sur les transformations de la médecine et des interventions préventives comme sur la médicalisation de la société, et même sur la consistance de la solidarité sociale. Dans cette perspective, on considérera alors le dépistage comme un bon « analyseur » des mutations qui travaillent nos sociétés.

Avec cette précision que si cet objet est heuristique, il reste cependant difficile à définir. Le dépistage est en effet un procédé polymorphe, mobilisé dans différents contextes institutionnels et avec des finalités variables. Qu'y a-t-il de commun, par exemple, entre le dépistage des troubles auditifs par la médecine scolaire, le dépistage néonatal de la mucoviscidose et le dépistage des mutations génétiques associées au cancer du sein ? Autrement dit, comment faire d'une pratique médicale hétérogène - et ce d'autant plus si on l'examine dans son épaisseur historique - un objet sociologique à peu près circonscrit ? Il semble à cet égard qu'au-delà de la diversité des pratiques de dépistage, on peut dégager quatre invariants qui en précisent la nature: a) d'abord, le dépistage est ciblé sur une pathologie dont on cherche la trace, à la différence des examens de routine à large spectre ou des check-up auxquels il a longtemps été associé; b) il vise à hâter et à faciliter le travail de diagnostic et suppose de devancer son destin médical en l'absence de symptômes ou d'urgence thérapeutique; c) il s'ensuit que le dépistage s'adresse à des personnes bien portantes et vient outiller cette «médecine de surveillance» [3] chargée de scruter la santé des populations saines; d) enfin, le dépistage ne produit rien d'autre qu'une information, un savoir classant qui départage et trie les individus sur la base de critères médicaux. Ciblage sur une pathologie, surveillance étendue, production d'une information médicale et ventilation des individus : tels sont a priori les principes qui délimitent le cadre général des opérations de dépistage par delà la profusion des pratiques. Ces principes définissent une sorte de grammaire du dépistage, une matrice commune dont il s'agit alors d'étudier les transpositions successives dans différents contextes sociaux, politiques et scientifiques.

C'est ce travail que l'on voudrait esquisser ici en soulignant, dans une perspective sociohistorique [4], certaines évolutions qui caractérisent les politiques de dépistage menées en France. On s'attachera ainsi à présenter trois politiques de dépistage qui traversent le 20ème siècle pour, ensuite, tenter d'éclairer, de manière plus générale et plus théorique, les transformations de l'État dépisteur contemporain.

\section{I- Le dépistage au fil de trois maux : tuberculose, can- cer, sida}

Les trois pathologies évoquées dans cette partie figurent assurément parmi les grands fléaux morbides du 20ème siècle. Mais ce qui justifie la comparaison à laquelle on voudrait se livrer ici, ce n'est pas seulement ce rôle de maladies emblématiques: c'est plutôt leur place dans l'histoire des techniques d'immunisation [5]. De fait, il n'y a initialement pas de vaccin pour les combattre, de sorte quetuberculose, cancer et sida ont, au départ, le même statut sur le plan immunologique. Pour chacune d'elles, alors, le dépistage va jouer un rôle crucial: il faut repérer la maladie le plus tôt possible pour endiguer sa progression. Or, les stratégies préventives qui sont successivement mises en place donnent à voir des évolutions importantes et suggestives.

La tuberculose, tout d'abord, est la principale « maladie sociale » à la fin du 19èmesiècle et au début du 20ème siècle, celle qui, avec la syphilis, va faire entrer le dépistage dans l'ère de la production de masse. Ce type de dépistages se développe à la faveur d'initiatives locales à une époque où l'État hygiéniste ne dispose pas encore, en France, des moyens de ses ambitions. Et la paternité en revient à bien des égards à Albert Calmette, le directeur de l'Institut Pasteur de Lille dès 1895. L'objectif de ce dernier est alors de « rechercher, attirer et retenir » tous ceux qui sont exposés à la tuberculose, et le dépistage consiste donc à débusquer les tuberculeux pour neutraliser le danger qu'ils représentent $[6,7]$. Plus exactement, le dépistage apparait à l'époque comme une opération ambiguë qui ne relève ni de la police - qui réprime - ni de l'assistance - qui soulage - mais les redéploye, l'une et l'autre, dans le cadre d'une stratégie inédite de défense sociale.

Sans entrer dans le détail du dispositif conçu par Calmette, on peut souligner que cette stratégie conduit à soumettre l'intérêt individuel à l'intérêt général. Calmette préconise en effet d'implanter dans les villes des dispensairesde prophylaxie, véritables commissariats sanitaires à partir desquels des "visiteurs ouvriers " auront pour mission de " dépister, sans être l'objet de la moindre crainte, les foyers tuberculeux dans les logements insalubres et les familles 
pauvres » [8]. Initialement, le dépistage de la tuberculose suppose donc de pénétrer l'intimité des familles ouvrières et de rabattre les cas suspects vers le dispensaire. Là, les individus sont examinés, enregistrés, immatriculés et questionnés sur leur mode de vie et leurs fréquentations, de manière à délimiter les zones d'infection - le « contage » - et à orienter les recherches futures. Le rôle du dispensaire, en réalité,est double. D'un côté, il s'efforce de trier les malades et de repérer les plus curables afin de les orienter vers les sanatoria, où le nombre de places est compté. Et d'un autre côté, il s'emploieà neutraliser les malades les plus dangereux, les « cracheurs de bacilles ", en établissant autour d'eux une véritable « barrière sanitaire » [9]. Pour ces malades privés de cure et laissés sur place, le personnel des dispensaires procède à la désinfection régulière des logements, lessive le linge souillé, distribue des crachoirs de poche et veille au respect scrupuleux des règles d'hygiène ne pas fumer, boire et balayer à sec, ne pas cracher par terre ni porter la barbe, entre autres prescriptions. Ce rôle de surveillance échoit en particulier au visiteur, que Calmette qualifie d'ailleurs de «moniteur » car il a en charge, à cet égard, une véritable orthopédie des comportements et peut " surgir à tout moment pour s'assurer que les conseils donnés sont exactement suivis » [6].

Pour les pasteuriens qui, comme Calmette, conçoivent ces premiers dispositifs de dépistage, le malade constitue avant tout une menace et, en tant que tel, « on a le droit de l'empêcher d'être nuisible à la communauté " [10]. Sous cet éclairage, ce type de dépistage s'apparente alors à ces «dispositifs immunitaires » qu'évoque le philosophe Roberto Esposito [11], des dispositifs qui, certes, assurent l'autoprotection de la société, mais qui engendrent toujours le rétrécissement ou la clôture de la communauté, en limitant les échanges, en emprisonnant les libertés ou en brisant les solidarités. N'est-ce pas ce qui se passe, en effet, avec ces dépistages qui fonctionnent comme de véritables check-points et hérissent la communauté de «barrières sanitaires»?

Cette allusion aux réflexions d'Esposito est stimulante car elle permet une comparaison fructueuse avec la gestion politique du sida, une maladie qui, justement, s'attaque aux défenses immunitaires des individus et présente, elle aussi, un caractère épidémique. Or, on constate que si des mesures de défense sociale ont été évoquées en France dans les années 1980, la politique mise en œuvre n'a pas été conçue pour repérer et identifier à tout prix les personnes séropositives [12].

Aux « dérives immunitaires » qui caractérisaient le dépistage de la tuberculose au début du 20ème siècle s'oppose une tout autre démarche à la fin du siècle dans la lutte contre le sida. La politique qui est impulsée s'exonère en effet des principes en vigueur pour la syphilis et les autres maladies transmissibles, à telle enseigne qu'on a pu parler de « l'exceptionnalisme du sida » [13]. Dans ce cadre, point de surveillance, de fichage, de sanction ou de tri, mais une gestion libérale de l'épidémie qui met l'accent sur la participation volontaire des individus aux opérations de dépistage, ce qui exclut des dépistages systématiques ou obligatoires. De fait, quels que soient la situation (examens prénataux ou prénuptiaux) et le contexte (hôpital, prison, armée, etc.), le dépistage du VIH-Sida requiert le consentement exprès des intéressés. On assiste, par conséquent, au triomphe d'une approche « éthique » fondée sur l'idée selon laquelle,en l'absence de toute riposte thérapeutique, dépister n'apporte aucun bénéfice à l'individu et n'a de sens, en réalité, que pour infléchir les comportements à risques, ce qui suppose la collaboration consciente des personnes dépistées. Avec le sida, autrement dit, les droits des individus sont devenus de véritables outils de santé publique [13] tandis que le « dispositif immunitaire » est finalement laissé à l'initiative de chacun. C'est là un renversement total par rapport aux modes de prise en charge de la tuberculose quelques décennies auparavant. Un renversement d'autant plus étonnant qu'à partir du milieu des années 1990, avec l'arrivée des trithérapies et une meilleure connaissance de la cinétique virale, on sait qu'un diagnostic et une prise en charge précoces augmentent l'efficacité des traitements tout en diminuant les risques de transmission - à un partenaire sexuel ou à un fœtus en gestation.

Comment expliquer, dès lors, l'apparition puis le maintien d'une politique fondée avant tout sur la responsabilisation des individus, une politique qui ne sera infléchie dans le sens d'une plus grande systématicité des tests qu'à partir du “ Plan Sida » de 2010 ? La réponse à cette question peut être trouvée en examinant ce qui s'est joué sur le front de la lutte contre le cancer $[14,15]$. Pendant longtemps, en effet, les médecins étaient convaincus que si, à la différence de la tuberculose, le cancer n'était pas évitable au moyen d'une prophylaxie sociale, il était néanmoins possible de le soigner. La radiothérapie pouvait ainsi être mobilisée, de même que la chirurgie, mais à la condition d'agir vite et de faire un « diagnostic précoce ». C'est ce qu'exprime par exemple le cancérologue Raymond Molinéry en 1928 :

« On ignore l'origine du mal. Il n'existe aucun remède spécifique pour le guérir ; mais ce qu'on sait, c'est qu'au début il est local. Si l'on extirpe ou si l'on détruit toutes les cellules malades, le cancer peut être supprimé et ne jamais réapparaître. Il faut donc agir vite, alors que le mal peut être enlevé radicalement. Mais, à son début, le cancer est généralement indolore et sans symptômes inquiétants. La première condition d'une lutte efficace est donc de préciser les premiers signes qui peuvent faire craindre un cancer et d'instruire le public pour qu'il consulte un médecin dès que ces signes paraissent » [16]. 
De fait, parce que le cancer est « indolore ", asymptomatique et, pour ainsi dire, furtif, on ne peut concevoir de tests spécifiques ni de dépistages étendus. Établir un « diagnostic précoce » suppose donc, comme l'écrit l'auteur, «d'instruire le public pour qu'il consulte un médecin » dès les premiers signes de la maladie. Mais encore faut-il, alors, que chacun soit en mesure de reconnaître ces signes cliniques et s'en alarme, que chacun, par conséquent, prête attention à corps et à son métabolisme. Autrement dit, si le diagnostic doit être posé par des médecins spécialisés, le dépistage devient ici l'affaire de tous [17]. Et comme le souligne Patrice Pinell [15], le dépistage du cancer, à son démarrage, réclame finalement des individus réflexifs, capables de transformer une inquiétude raisonnée en vigilance active, des individus-dépisteurs, en somme, aptes à l'auto-surveillance et susceptibles de devenir les auxiliaires des professionnels de santé. Or, tout porte à croire que c'est justement cet individu autonome et responsable, propulsé sur le devant de la scène médicale par la lutte anticancéreuse, que convoquera, plus tard, la politique de prévention du VIH-Sida.

\section{II- Métamorphoses de l'État dépisteur}

Ce que suggère cette séquence tuberculose-cancer-sida, c'est une évolution assez nette des pratiques et politiques de dépistage, une rupture historique qu'éclairent en particulier les analyses du philosophe Michel Foucault.Celui-ci souligne en effet avec force combien, dans la période moderne, le corps est saisi par des savoirs - biologie, démographie, médecine, etc. - et fait l'objet d'une administration méthodique par un pouvoir qui se donne pour fonction de surveiller, d'inciter et de majorer la vie et la santé. Le pouvoir n'est plus alors un instrument de " soustraction économique», un pouvoir « percepteur et prédateur » [18] : il investit le corps des individus sous la forme d'un « biopouvoir» dont le dépistage, tel que le conçoivent les pasteuriens au début du 20ème siècle, est à la fois l'instrument et l'expression. De fait, le dispositif imaginé par Calmette, puis reproduit dans de nombreuses agglomérations françaises, est emblématique de cette logique foucaldienne. Cette logique est celle d'un gouvernement autoritaire des conduites individuelles ; elle suppose une emprise directe sur les corps, doublée d'une action normalisatrice, et implique l'enrôlement des individus, sans ménagement, dans un système de contrôles et de contraintes. Or, de ce point de vue, il y a bien une discontinuité radicale entre cette forme de « gouvernement des corps ", qui est aussi un gouvernement de la misère, et les stratégies préventives qui lui ont succédé, dans le cadre desquelles le dépistage est davantage l'instrument d'un bon gouvernement de soi.

Comment, dès lors, expliquer ce basculement ? Comment comprendre cet effacement apparent du « biopouvoir » ? Il s'agit là, assurément, d'un changement de logique dont le déroulement historique est lent et complexe. Néanmoins, deux dynamiques sociales permettent de rendre compte de cette métamorphose de l'État dépisteur contemporain.

La première dynamique est celle que pointe Patrice Pinell $[14,15]$ : l'histoire du dépistage accompagne ce que le sociologue Norbert Elias a appelé le «processus de civilisation», un processus par lequel les individus intériorisent peu à peu les contraintes sociales, rationalisent leur conduite et se conforment spontanément aux exigences de la vie en commun [18]. Ce processus historique permet de comprendre qu'à une logique " foucaldienne » se substitue, en matière de prévention et de dépistage, une logique "éliasienne " fondée sur l'intériorisation des contraintes sanitaires - qui deviennent donc des autocontraintes - et sur l'autogestion, par chaque individu, de son destin corporel. Dans cette perspective, la « barrière sanitaire » que les pasteuriens rêvaient d'établir autour des malades contagieux devient moins nécessaire : elle est remplacée par ce « mur invisible » [18] que la civilitéa dressé entre les corpset qui les isole à mesure que chacun acquiert une conscience de soi plus aiguë.

On peut d'ailleurs suggérer que c'est là le rêve de la « médecine de surveillance » contemporaine : l'intériorisation des impératifs sanitaires par des individus dociles et responsables qui deviennent les auxiliaires du «biopouvoir » et parachèvent le processus d'immunisation sociale [3]. De fait, les opérations de dépistage ne s'adressent pas tant, désormais, à des individus qu'il faudrait surveiller qu'à des individus façonnés par le «processus de civilisation » et jouant spontanément le jeu de la surveillance. La commercialisation des autotests en matière de $\mathrm{VIH}$-sida traduitpar exemple cette inflexion en déléguant à l'individu le contrôle de son statut sérologique. Avec cependant cette précision qu'il y a loin du rêve à la réalité en matière de surveillance médicale.Car cet individu responsable de sa santé, à la fois autonome et obéissant, est en fait assez rare, particulièrement au bas de la stratification sociale. On entrevoit là, finalement, une évolution assez singulière : ceux-là mêmes qui, au début du siècle dernier, payaient du prix de la discrimination leur prise en charge par les missionnaires de l'hygiène publique passent souvent au travers d'une politique devenue aujourd'hui moins autoritaire.

La seconde dynamique, solidaire de la précédente, est celle de la démocratisation de la société, qui commande de revoir les conditions de l'intervention sanitaire. La révolution égalitaire, en particulier, va peu à peu condamner toute forme d'exclusion ou de discrimination, dans le champ de la santé comme ailleurs. Cette dynamique puissante est celle dont rendent compte notamment les travaux de Marcel Gauchet et Gladys Swain sur ce cas-limite qu'est l'institution asilaire dans la France du 19ème siècle. Pour ces auteurs, l'asile 
incarne en réalité les contradictions des dispositifs autoritaires de l'État à l'âge démocratique. Ils montrent en effet que l'enfermement des fous n'est concevable que dans une société où coexistent des mondes séparés, c'est-à-dire des individus considérés comme relevantd' « espèces différentes». Mais il est incompatible avec l'esprit démocratique et va dès lors changer de sens à partir du 19ème siècle : si les fous sont alors encasernés et soustraits au monde, ils s'inscrivent néanmoins dans un projet de réinsertion au sein des circuits de communication et d'échange intra-asilaires [19].

Or, ce qui se joue ainsi au cœur même de cette « institution totale " qu'est l'asile illustre avec éclat un processus qui, en réalité, travaille en profondeur tous les compartiments de la vie sociale et de l'action publique. Carl'existence d'un «monde commun » démocratique, qui dissout l'altérité et atténue peu à peu les hiérarchies instituées, exige en fait de l'État qu'il ajuste sans cesse ses interventions pour produire de l'égalité. Égalité des droits, d'abord ; égalité des conditions, ensuite - celle que revendiquent par exemple ces mouvements de femmes qui, dans les années 1970 encore, réclament de pouvoir toutes bénéficier des techniques de dépistage et de diagnostic du cancer du sein [20]. Mais aussi égale dignité des individus qui condamne, dans tous les domaines, les dispositifs susceptibles de stigmatiser tel ou tel groupe. C'est cette même dynamique qui accompagne aujourd'hui la montée en puissance des droits subjectifs, ces « droits des individus » qui tendent à faire prévaloir l'autonomie des personnes sur les nécessités de la vie collective [21]. En France, la refonte, en 2000, des dispositions du Code de la santé publique relatives à la lutte contre les « fléaux sociaux » et l'encadrement législatif des pratiques de dépistage reflètent assez bien cette tendance, de même que le thème des « droits du malade » qu'inaugure, un siècle après celle de 1902, la nouvelle grande loi sanitaire française. Plus question, dès lors, de dépister les individus sans leur consentement, du moins théoriquement ; plus question, non plus, d'enfermer les malades contagieux sans autre forme de procès ni de les soumettre à des contrôles inopinés ou intempestifs tels que les pratiquait le « visiteur » de Calmette. De fait, la sensibilité égalitaire actuelle suppose de plus en plus d'installer les individus en situation de choisir pour euxmêmes, en situation de participer et de s'impliquer, y compris dans les dispositifs de prévention.

\section{Conclusion}

L'histoire des pratiques de dépistage est assurément marquée par ce double mouvement : l'avancée de la civilité et l'approfondissement de la démocratie. Pour autant, il ne faut peut-être pas conclure trop vite à une acclimatation démocratique des dépistages, avec un adoucissement des contraintes normalisatrices et une lente affirmation des libertés individuelles. Car si l'on assiste sans doute à un assouplissement des formes les plus immédiates du « gouvernement des corps ", on peut cependant s'interroger sur la possible émergence d'un gouvernement par les corps : un corps qui ne ment pas et qui livre ses secrets à une société avide de sécurité. Ce disant, il ne s'agit évidemment pas de déplorer le développement des dépistages car ceux-ci demeurent indispensables, en France, en Algérie et dans la plupart des pays, notamment pour combattre les grands fléaux morbides comme la tuberculose [22]. En revanche, on voudrait souligner l'enjeu que représentent les données de santé issues des dépistages à une époque où l'on peut aisément stocker les informations et croiser les fichiers. Ce qui est en jeu ici, ce n'est pas seulement le degré de confidentialité des données médicales - même si cette préoccupation est bien sûr essentielle. Le problème plus fondamental que soulèvent en l'espèce la multiplication et la banalisation des dépistages peut se formuler ainsi : quelle place faut-il faire à la dimension corporelle de l'individu dans une société démocratique (Esposito, 2010) ? En d'autres termes, une société reposant sur l'égalité des citoyens peut-elle s'accommoder d'une connaissance de plus en plus fine de leurs différences, d'une « transparence du mal » pour parler comme Jean Baudrillard?

\section{Conflits d'intérêt}

L'auteur déclare n'avoir aucun conflit d'intérêt.

\section{Références bibliographiques}

[1] Nelkin Dorothy, Tancredi Laurence, 1989, Dangerous diagnostics. The social power of biological information, New York, Basic Books.

[2] Sicard Didier, 2002, La médecine sans le corps. Une nouvelle réflexion éthique, Paris, Plon.

[3] Armstrong David, 1995, "The Rise of Surveillance Medicine", Sociology of Health and Illness, vol.17, n³, p.393-404.

[4] Castel Robert, 1997, « Présent et généalogie du présent : une approche non évolutionniste du changement ", dans Coll., Au risque de Foucault, Paris, Éditions du Centre Pompidou, p.161-168.

[5] Moulin Anne-Marie, 1991, Le dernier langage de la médecine. Histoire de l'immunologie de Pasteur au Sida, Paris, PUF.

[6] Calmette Albert, 1901, "Le dispensaire antituberculeux Émile Roux à Lille ", Revue d'hygiène et de police sanitaire, $n^{\circ} 23$, p. 577 598.

[7] Calmette Albert, 1909, « Le préventorium ou dispensaire et son rôle dans la lutte antituberculeuse ", dans Coll., Lille et la région du Nord en 1909, Lille, Imprimerie Danel, tome 1, p.1047-1051.

[8] Rénon Louis, 1905, Les maladies populaires, Paris, Masson et Cie.

[9] Courmont Jules, 1905, « Le dispensaire antituberculeux », Lyon 
Médical, 37ème année, tome 54, p.377-383.

[10] Duclaux Émile, 1902, L’hygiène sociale, Paris, Félix Alcan.

[11] Esposito Roberto, 2010, Communauté, immunité, biopolitique Paris, Les prairies ordinaires.

[12] Dodier Nicolas, 2003, Leçons politiques de l'épidémie de sida, Paris, Éditions de l'EHESS.

[13] Heard Mélanie, 2007, Un nouveau paradigme en santé publique : droits individuels et VIH/Sida, 25 ans d'action publique en France, Thèse de science politique, IEP de Paris.

[14] Pinell Patrice, 1992, Naissance d'un fléau. Histoire de la lutte contre le cancer en France (1890-1940), Paris, Métailié.

[15] Pinell Patrice, 1996, "Modern medicine and the civilising process", Sociology of Health and Illness, vol.18, n¹, p.1-16.

[16] Molinéry Raymond, 1928, « L'armement anticancéreux, où en sommes-nous ? ", La médecine internationale, $n^{\circ}$ 4, p. 129-134.
[17] Oberling Charles, 1954, Le cancer, Paris, Gallimard.

[18] Foucault Michel, [1981] 2001, " Les mailles du pouvoir », dans Dits et Ecrits II, Paris, Quarto-Gallimard, p.1001-1021.

[18] Elias Norbert, [1939] 1973, La civilisation des mœurs, Paris, Agora Pocket.

[19] Gauchet Marcel, Swain Gladys, [1980] 2004, La pratique de l'es prit humain. L'institution asilaire et la révolution démocratique, $\mathrm{Pa}$ ris, Gallimard-NRF.

[20] Löwy Ilana, 2010, PreventiveStrikes. Women, Precancer, and Prophylacticsurgery, Baltimore, The Johns Hopkins UniversityPress.

[21] Schnapper Dominique, 2002, La démocratie providentielle. Essai sur l'égalité contemporaine, Paris, Gallimard-NRF.

[22] Snouber Abdelmadjid, 2014, «Quelles solutions pour contenir le cours évolutif de la problématique tuberculose en Algérie ? ", Les Cahiers du GRAS, n³, p. 78-107. 